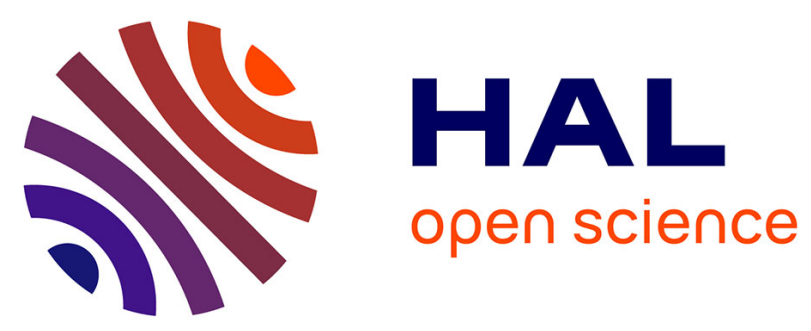

\title{
Effects of dual tasking on postural and gait performances in children with cerebral palsy and healthy children
}

\author{
Estelle Palluel, Guillaume Chauvel, Véronique Bourg, Marie-Christine
} Commare, Chloé Prado, Vincent Farigoule, Vincent Nougier, Isabelle Olivier

\section{- To cite this version:}

Estelle Palluel, Guillaume Chauvel, Véronique Bourg, Marie-Christine Commare, Chloé Prado, et al.. Effects of dual tasking on postural and gait performances in children with cerebral palsy and healthy children. International Journal of Developmental Neuroscience, 2019, 79 (1), pp.54-64. 10.1016/j.ijdevneu.2019.10.008 . hal-03216921

\section{HAL Id: hal-03216921 \\ https://hal.science/hal-03216921}

Submitted on 4 May 2021

HAL is a multi-disciplinary open access archive for the deposit and dissemination of scientific research documents, whether they are published or not. The documents may come from teaching and research institutions in France or abroad, or from public or private research centers.
L'archive ouverte pluridisciplinaire $\mathbf{H A L}$, est destinée au dépôt et à la diffusion de documents scientifiques de niveau recherche, publiés ou non, émanant des établissements d'enseignement et de recherche français ou étrangers, des laboratoires publics ou privés. 
1 Running Head: Dual tasking in CP and healthy children

2

3 Effects of dual tasking on postural and gait performances in children with cerebral palsy and healthy children

Estelle Palluel ${ }^{1}$

Guillaume Chauvel ${ }^{2}$

Véronique Bourg ${ }^{3}$

Marie-Christine Commare ${ }^{3}$

Chloé Prado 3

Vincent Farigoule ${ }^{3}$

Vincent Nougier ${ }^{1}$

Isabelle Olivier ${ }^{1}$

$15{ }^{1}$ Univ. Grenoble Alpes, CNRS, TIMC-IMAG, F-38000 Grenoble, France

$16{ }^{2}$ Univ. Paris Est Creteil, LIRTES, 94009 Creteil cedex, France

$17{ }^{3}$ Department of Physical Medicine and Readaptation, University Hospital Grenoble-Alpes,

18 University Grenoble-Alpes, France

19

Corresponding Author: Estelle Palluel

Université Grenoble Alpes

38706 La Tronche Cedex, France 
Abstract

Simultaneous execution of motor and cognitive tasks is embedded in the daily life of

27 children. 53 children of 7-12 years and 22 adults (study 1), 20 healthy children and 20 children of

28 7-12 years with cerebral palsy (study 2) performed a Stroop-animal task simultaneously with a

29 standing or a walking task in order to determine the attentional demand of postural control and

30 locomotion. Dual-task cost decreased with advancing age in healthy children during balance. CP

31 and healthy children were similarly affected by dual-task constraints during standing and

32 walking. Children with diplegia were more affected by the DT during the postural task than

33 children with hemiplegia. We found that adults could benefit from dual-tasking for standing. The

34 integrated model of task prioritization might explain our results regarding postural reserve of

35 each population.

36

37 Keywords: dual-tasking; posture; gait; Stroop; children; cerebral palsy

Word count: 6129 words

What this paper adds:

42 - Dual Task Cost decreases with advancing age in healthy children during balance and

$43 \quad$ adults could benefit from dual-tasking for standing

$44 \quad$ - CP and healthy children were similarly affected by dual-task constraints during standing

$45 \quad$ and walking

46 - Children with diplegia were more affected by the DT during the postural task than children with hemiplegia 


\section{Introduction}

Many motor tasks such as standing and walking are often performed simultaneously with

51 a cognitive task (e.g. talking, looking for a building). Even though the control of posture and gait

52 is traditionally considered as automatic, several studies using dual-task paradigms demonstrated a

53 clear link between the regulation of sway or locomotion and higher-order processes ${ }^{1-5}$. In healthy

54 adults, an improvement of standing has been reported with simple cognitive tasks ${ }^{6-8}$. These

55 cognitive tasks withdraw participants' attention from monitoring their postural performance. This

56 in turn, allows the motor system to function in an unconstrained mode ${ }^{9}$ and promotes automatic

57 processes for regulating posture ${ }^{10}$ and gait ${ }^{11}$. However, some studies showed no effect ${ }^{12,13}$ or a

58 deterioration of standing ${ }^{14,15}$ whereas most studies reported a deterioration of gait in dual-tasking $59 \quad 4,16$.

Three models are currently used to explain these apparently contradictory results: The

61 cross-domain competition model, the U-shaped non-linear interaction model and the task

62 prioritization model ${ }^{17}$. In the cross-domain competition model, the limited attentional and

63 processing capacity leads to a division of and thus a competition for the attentional resources

64 between the cognitive and postural tasks. This model can only explain why increasing the

65 difficulty of the cognitive (or postural) task systematically involves a degradation of postural (or

66 cognitive) performance. The performance decrement of one or both tasks is known as cognitive-

67 motor interference $\left(\mathrm{CMI} ;{ }^{18}\right)$ or dual-task cost $\left(\mathrm{DTC} ;{ }^{19}\right)$. The U-shaped non-linear interaction

68 model suggests that the performance of an easy cognitive task and /or a task with an external

69 focus of attention can shift the focus of attention away from postural control and leads to a better

70 postural control relative to a single-task baseline. However, increasing the difficulty of the

71 cognitive task can result in a degradation of postural sway because of the competition of

72 attentional resources already described in the cross-domain competition model ${ }^{6}$. Finally, the task 
73 prioritization model postulates that subjects prioritize postural control or locomotion over

74 cognitive activity under specific conditions (e.g., postural threat conditions). This "posture first

75 principle" has been particularly observed in the elderly ${ }^{20}$ or in patients exhibiting vestibular

76 disorders ${ }^{21}$. Yogev-Seligmann et al. ${ }^{22}$ introduced the "integrated model of task prioritization"

77 and explained that different self-selected strategies can be handled in dual-tasking: "posture/gait-

78 first" or "posture/gait-second" strategies depend on postural reserve and hazard estimation.

79 People with high postural reserve and hazard estimation are able to prioritize the cognitive task

80 during an easy motor task. If this latter becomes more complex, they allocate more resources to

81 the posture/gait task and avoid putting their balance at risk.

Postural stability, walking performances and attentional resources increase with age. A

83 turning-point has been observed around 7-8 years of age for standing ${ }^{23,24}$. More precisely, an

84 improvement of gait parameters during childhood, with an increase of velocity $(60-110 \mathrm{~cm} / \mathrm{s})$

85 and stride length $(40-90 \mathrm{~cm})$ has been observed between 1 and 7 years ${ }^{25}$. Characteristics of

86 walking become steady and similar to the adults' pattern around 7-8 years of age ${ }^{26}$. However,

87 temporal and spatial parameters can still improve around 11-15 years, which can be explained by

88 the continuing development of the nervous system and the changes of the anthropometric

89 characteristics ${ }^{27}$. During childhood, some inconsistent results in dual-task conditions have also

90 been obtained in postural control ${ }^{28}$ and walking performances ${ }^{29-31}$. Olivier et al. ${ }^{8}$ showed an

91 improvement of standing in children aged 4-11 years while watching a movie. Schaefer el al. ${ }^{31}$

92 reported an increase of gait regularity in children aged 7 and 9 years. Hagman-von Arx ${ }^{32}$

93 reported that 6-13 years children were more affected by a motor dual-task condition (i.e., fasten

94 and unfasten a shirt button while walking) than by a cognitive dual-task situation (i.e., listening

95 and memorizing digits while walking). Boonyong et al. ${ }^{33}$ observed a decrease of gait speed and

96 step length when avoiding an obstacle during an auditory Stroop-task. They suggested a 
97 developmental trend in attentional resources used to control gait in typical children. Most of these 98 results illustrated the cross-domain competition model.

99 Research on children with cerebral palsy (CP) shows a poor balance control and muscular

100 weakness ${ }^{34}$. CP can be distinguished as (1) tetraplegia affecting all four limbs; (2) diplegia, with

101 lower limbs more compromised than upper ones; (3) hemiplegia, affecting only one side of the

102 body; and (4) monoplegia, altering a single limb ${ }^{35}$. These children often experience ankle, knee

103 and hip stiffness that result in an atypical posture such as the crouched posture during stance.

104 Children with unilateral spastic CP (spastic hemiplegia) tend to have an asymmetric alignment

105 with their weight displaced toward the non-affected side in order to compensate for weakness in

106 the hemiparetic leg. Hemiplegic gait consists of equinus and intoeing with crouch (i.e. increased

107 knee flexion on the hemiplegic side) in stance and a stiff knee in swing in approximately half of

108 the subjects in the study of Wren et al. ${ }^{36}$. Diplegic gait can be characterized as stiff-kneed,

109 crouched, and intoed, with excessive hip flexion and equinus ${ }^{36}$. Crouch is generally more

110 prominent in children with diplegia. Previous findings reported deleterious effects of time on the

111 walking ability of children with CP with for example an increasing likelihood of joint deformities

112 such as a rotational misalignment between the femur and tibia ${ }^{37,38}$. Gait impairments include

113 slower speed, reduced step length, wider step width ${ }^{39}$. Additional impairments include deficits

114 in sensory and cognitive functions such as visuospatial attention ${ }^{40}$. CP children exhibit greater

115 dual-task interference during standing and walking while performing a secondary cognitive task

$116{ }^{41-44}$. Donker et al. ${ }^{45}$ described an increased Center of Pressure regularity in CP children that

117 might be interpreted as the dynamical signature of a poor postural control.

118 Within this complex context, two complementary studies were conducted to investigate

119 the typical and atypical development of standing and walking under dual-task conditions. In 120 study 1, we explored the effects of an adapted Stroop task for children (see Methods for details) 
121 on posture and gait by comparing younger healthy children aged 7-9 years and older healthy

122 children aged 10-12 years with adults. In study 2, we applied the same protocol to CP and age-

123 matched healthy children. We hypothesized that postural performances should improve in adults

124 in the dual-task condition because of the low difficulty level of the cognitive task. However, it

125 should impair 1) postural sway in healthy and CP children and 2) gait in all populations ${ }^{3}$. Due to

126 impairments in both the postural control and executive attention network systems, we also

127 predicted that CP children and especially children with diplegia would be more affected by dual-

128 task constraints than healthy children. The expected results should confirm the "integrated model

129 of task prioritization "and have potential clinical applications for CP children rehabilitation.

\section{Method}

132 Participants

133 children, divided in two age groups: $7-9$ years $(10$ girls and 15 boys; mean age $=7.9$

134 years \pm 0.8 ), 10-12 years ( 11 girls and 17 boys; mean age $=10.9$ years \pm 0.8$)$ and 22 adults aged

$13518-32$ years (13 females and 9 males; mean age $=22.1$ years \pm 5.5$)$ took part in the first study.

136 Children were recruited from a sport and recreational centre and adults were recruited from the

137 local university and from surrounding local communities.

13820 children with cerebral palsy (CP) and 20 healthy age-matched children that were selected

139 from study 1 participated in the second study. They were divided in two age groups: 7-9 years (2

140 females and 8 males for $\mathrm{CP}$ children; mean age $=8.5$ years $\pm 0.7 ; 2$ females and 8 males for healthy 141 children; mean age $=8.2$ years \pm 0.9$)$ and $10-12$ years $(5$ females and 5 males for CP children; mean 142 age $=10.4$ years $\pm 0.8 ; 5$ females and 5 males for healthy children; mean age $=10.3$ years \pm 0.8 ) 143 Children with CP were referred for participation from the Grenoble University Hospital. $11 \mathrm{CP}$ 144 children were diplegic and 9 were hemiplegic. All of them were at the first level of the Gross Motor 
145 Classification System. No exclusion criteria were specified regarding treatments, but none of the

146 children had surgery or botulinum toxin injections within three months before the assessment.

147 All participants had normal or corrected to normal vision, no auditory deficits, and had no

148 history of psychiatric disorders. No neurological disorders were reported for adults and healthy

149 children. They were naïve to the purpose of the study and gave written informed consent as

150 required by the Declaration of Helsinki (1964). The study was approved by the ethics research

151 committee (n²012-A00710-43) and parents' agreement was also obtained for the children.

152

153 Apparatus, procedure and data analysis

$154 \quad$ Postural task

155 Participants stood barefoot on a force platform (OR6-AMTI1 ${ }^{\circledR}$ ) with feet abducted at $30^{\circ}$, 156 with the heels $7 \mathrm{~cm}$ apart and arms positioned along the body in order to maintain a comfortable

157 and relaxed posture. The feet position was marked on the platform to ensure the reproducibility

158 of the position. Three 30s-trials per condition were performed and all conditions were

159 randomized. Participants were asked to stand as still as possible.

160 Antero-posterior (AP) and medio-lateral (ML) displacements of the Centre of foot

161 Pressure (CoP) were recorded. Data were collected at a frequency of $100 \mathrm{~Hz}$ and filtered with a 162 second-order Butterworth filter with a cut-off frequency of $10 \mathrm{~Hz}$. Five dependent variables of 163 CoP displacements were calculated: The mean amplitude on the AP and ML axes (in mm), the 164 mean speed on the AP and ML axes (in $\mathrm{mm} / \mathrm{s}$ ) and the global sample entropy to evaluate the 165 regularity of postural sway. Sample entropy was determined using the method developed by 166 Richman and Moorman ${ }^{46}$. As previously suggested, parameters values were $m=3$ (number of 167 data points to compare) and $\mathrm{r}=0.4$. 
Walking task

170 Children and adults were instructed to walk without shoes over a $5.20 \mathrm{~m}$ long electronic

171 walkway $\left(\right.$ Gaitrite $\left.{ }^{\circledR}\right)$ at a self-paced speed. They started and finished walking $2.50 \mathrm{~m}$ before and

172 after the walkway. A screen $(200 \mathrm{~cm} \times 150 \mathrm{~cm})$ was placed $3 \mathrm{~m}$ after the walkway. Three trials

173 per condition were performed and all conditions were randomized. In this task, data from four

174 adults had to be discarded because of technical data collection problems: A total of 18 adults

175 were thus included.

176 Relevant temporal and spatial gait parameters were calculated by the Gaitrite software ${ }^{3}$.

177 Six parameters were analysed: Four temporal parameters - mean speed, cadence, step duration,

178 and percentage of double limb support duration with respect to gait cycle duration - and two

179 spatial parameters - normalized step length and normalized base of support, with respect to

180 participants' limb length.

181

Cognitive task

A dual-task paradigm, in which participants performed a Stroop task (DUAL task

184 condition: DT) simultaneously with the postural or the walking task was used to manipulate the 185 attentional demand of these motor tasks. It was a computerized version of an animal-Stroop test

186 in which participants were instructed to name the body of the animal that appeared on the screen:

187 Cow, duck, rabbit, sheep and pig. Stroop-like interference was elicited when the animal's head

188 did not correspond to its body. Participants were required to name the animal as quickly and as

189 accurately as possible with reference to the body and inhibit a preferred response based on

190 identification of the animal's head ${ }^{47}$. The animals were always presented one by one on the

191 screen and the following animal was immediately presented once participants had responded.

192 Warm-up trials were used to introduce the task. 
In a control condition (SINGLE task condition: ST), participants fixated a cross located at

194 the centre of a white $200 \mathrm{~cm}$ x $150 \mathrm{~cm}$ screen that was placed $3 \mathrm{~m}$ after the walkway. Participants

195 were asked to focus on the screen without moving and could rest whenever necessary between

196 postural and walking trials. The single and dual tasks were presented randomly among

197 participants in order to rule out presumable sequence effects.

198 Postural and walking dual-task costs (DTC) were estimated using the following equation

199 19: DTC $($ in $\%)=\frac{S T-D T}{S T} \times( \pm) 100$ where "ST" represents participant's motor performance in the

200 ST condition and "DT" represents participant's motor performance in DT condition. Positive

201 values indicated performance improvements, whereas negatives values indicated performance

202 impairments from single task to dual task. We used the $(+)$ multiplier for variables showing an

203 improvement with lower values in the dual- than single-task condition (i.e. CoP mean amplitude

204 and speed, step duration, normalized base of support and percentage of double limb support) and

205 the (-) multiplier for variables showing an improvement with higher values in the dual- than

206 single-task condition (i.e. sample entropy, mean speed, cadence, normalized step length). The

207 DTC was calculated for each variable. The mean standing/ walking DTC represented the average

208 of the standing/walking data and was a general indicator to compare the postural and walking

209 attentional cost with a similar cognitive task.

210

211 Statistical analyses

212 In study 1 , each dependent variable was submitted to a 3 age groups $(7-9,10-12$, and adult 213 groups) $\mathrm{x} 2$ attentional conditions (ST and DT conditions) analysis of variance (ANOVA) with

214 repeated measures on the last factor. We compared the mean DTC during the postural and the 
215 walking tasks with a 3 age groups (7-9, 10-12, and adult groups) x 2 tasks (postural and walking)

216 ANOVA with repeated measures on the last factor.

217 In study 2, each dependent variable was submitted to a 2 populations (CP and healthy) $\mathrm{x} 2$

218 age groups (7-9, 10-12 years) x 2 attentional conditions (ST and DT conditions) ANOVA with

219 repeated measures on the last factor. We compared the mean DTC during the postural and the

220 walking tasks with a 2 populations (CP and healthy) x 2 age groups (7-9, 10-12 years) x 2 tasks

221 (postural and walking) ANOVA with repeated measures on the last factor. A complementary

222 analysis was performed between children with diplegia and hemiplegia in study 2 to see whether

223 differences could be observed in these populations. No age effect was found across all measures.

224 We merged therefore the 7-9 years and 10-12 years groups in order to increase the power of the

225 analysis. We ran statistical analysis on $11 \mathrm{CP}$ children with diplegia (5 girls and 6 boys; mean age

$226=9.3$ years \pm 1.3 ) and 9 children with hemiplegia ( 2 girls and 7 boys; mean age $=9.7$ years \pm 1.2 )

227 regardless of the age but with a comparable group mean age. Each dependent variable was

228 submitted to a 2 deficits (diplegia and hemiplegia) x 2 attentional conditions (ST and DT

229 conditions) analysis of variance (ANOVA) with repeated measures on the last factor.

230 Post hoc analyses (Newman-Keuls) were used whenever necessary to determine specific

231 differences between populations, deficits (study 2), age groups and attentional conditions. As

232 interactions were the main expected results, they were presented first, followed by the main

233 effects of population and deficit (study 2), age and attentional condition. The Shapiro-Wilk tests

234 previously confirmed the normality of data. Eta squared values $\eta_{p}^{2}$ were also calculated for each

235 significant effect. The $\alpha$ level of significance was set at 0.05 .

237 Results 
240 children and an improvement of postural stability in adults during the DT condition were

241 observed. Statistical analysis revealed a two-way interaction of age group $\mathrm{x}$ attentional task for

242 AP and ML mean amplitude $\left(\mathrm{F}_{2,72}=3.70 ; p=0.030 ; \eta_{p}^{2}=0.09\right.$ and $\mathrm{F}_{2,72}=4.37 ; p=0.016 ; \eta_{p}{ }^{2}=0.11$, 243 respectively), $\mathrm{AP}$ and $\mathrm{ML}$ mean speed $\left(\mathrm{F}_{2,72}=4.29 ; p=0.017 ; \eta_{p}^{2}=0.11\right.$ and $\mathrm{F}_{2,72}=3.31 ; p=0.042$;

$244 \eta_{p}{ }^{2}=0.08$, respectively $)$ and sample entropy $\left(\mathrm{F}_{2,72}=6.66 ; p=0.002 ; \eta_{p}^{2}=0.16\right)$. Post-hoc analyses

245 showed an increase of AP mean amplitude (almost significant $p=0.051$ ), AP and ML mean speed

$246(p<0.001$ and $p=0.003$, respectively) in 7-9 years children during the DT condition. Unlike the

247 younger children, adults exhibited a decrease of AP and ML mean amplitude (almost significant

$248 p=0.054$ and $p=0.005$, respectively) and an increase of sample entropy $(p<0.001)$ during the DT

249 condition. AP and ML mean amplitude was smaller in adults and in 10-12 years children than in $2507-9$ years children during the ST $(p<0.01)$ and DT conditions $(p<0.001)$. AP and ML speed was

251 also smaller in adults than in 7-9 years children during the ST $(p<0.01)$ and DT conditions

$252(p<0.001)$. ML amplitude was smaller in adults than in 10-12 years children in the DT condition

$253(p<0.001)$. This difference was also significant for the AP and ML mean speed during the DT $254 \quad(p<0.05)$ and ST conditions $(p<0.01)$.

255 There was a main effect of age group for AP and ML mean amplitude $\left(\mathrm{F}_{2,72}=18.58\right.$;

$256 p<0.001 ; \eta_{p}{ }^{2}=0.34$ and $\left.\mathrm{F}_{2,72}=20.58 ; p<0.001 ; \eta_{p}{ }^{2}=0.36\right)$, and AP and ML mean speed $\left(\mathrm{F}_{2}\right.$,

$257 \quad 72=24.75 ; p<0.001 ; \eta p^{2}=0.41$ and $\left.\mathrm{F}_{2,72}=31.65 ; p<0.001 ; \eta p^{2}=0.47\right)$. A main effect of attentional

258 condition was found for AP mean speed $\left(\mathrm{F}_{1,72}=17.68 ; p<0.001 ; \eta p^{2}=0.20\right)$.

259 Adults showed a greater sample entropy in the DT than ST condition $(p<0.001)$. Sample

260 entropy was greater in adults than in 7-9 years $(p<0.001)$ and $10-12$ years $(p<0.01)$ children in the

261 DT condition, only. Main effects of age group and attentional condition were found for this 
262 variable $\left(\mathrm{F}_{2,72}=4.02 ; p=0.022 ; \eta^{2}=0.10\right.$ and $\left.\mathrm{F}_{1,72}=14.98 ; p<0.001 ; \eta^{2}=0.17\right)$. No other

263 interactions or main effects were significant.

Walking performances. As illustrated in Figure 2, an overall degradation of walking

performances during the DT condition was observed. 7-9 years children seemed more affected by

the dual-task than older children and adults. Statistical analysis revealed a two-way interaction of age group $\times$ attentional task for normalized step length $\left(\mathrm{F}_{2}, 68=5.04 ; p=0.009 ; \eta p^{2}=0.13\right)$ and normalized base of support $\left(\mathrm{F}_{2}, 68=3.57 ; p=0.033 ; \eta^{2}=0.09\right)$. Post hoc analyses revealed that

270 normalized step length was lower in the DT than ST condition for the 7-9 years, 10-12 years

271 children and adults $(p<0.001)$. The values were almost significantly different in the ST condition

272 between the 7-9 years children and the adults $(p=0.058)$. Normalized base of support was higher

273 in the DT than ST condition at 7-9 years $(p<0.001)$ and was higher during the DT condition in 7-

2749 years children than in 10-12 years children $(p=0.022)$ and adults $(p=0.010)$.

275 We found a main effect of age group for mean speed $\left(\mathrm{F}_{2}, 68=7.36 ; p=0.001 ; \eta p^{2}=0.18\right)$, 276 cadence $\left(\mathrm{F}_{2}, 68=8.55 ; p<0.001 ; \eta p^{2}=0.20\right)$, normalized base of support $\left(\mathrm{F}_{2}, 68=3.93 ; p=0.024\right.$

$\left.277 \eta_{p}{ }^{2}=0.10\right)$ and percentage of double limb support $\left(\mathrm{F}_{2}, 68=4.78 ; p=0.011 ; \eta^{2}=0.12\right)$. Post hoc 278 analyses showed that mean speed was significantly higher in adults than in 7-9 years $(p<0.001)$

279 and 10-12 years children $(p=0.019)$. Cadence was lower in adults than in $7-9$ years $(p<0.001)$ and 280 10-12 years children $(p=0.012)$. Percentage of double limb support was higher in adults than in 72819 years $(p=0.006)$ and $10-12$ years children $(p=0.016)$.

282 A main effect of attentional task was observed for mean speed $\left(\mathrm{F}_{1,68}=102.99 ; p<0.001\right.$;

$\left.283 \eta_{p}{ }^{2}=0.60\right)$, cadence $\left(\mathrm{F}_{1,68}=20.37 ; p<0.001 ; \eta_{p}{ }^{2}=0.23\right)$, step duration $\left(\mathrm{F}_{1,68}=13.49 ; p<0.001\right.$;

$\left.284 \eta_{p}{ }^{2}=0.17\right)$, normalized step length $\left(\mathrm{F}_{1,68}=159.79 ; p<0.001 ; \eta_{p}{ }^{2}=0.70\right)$, normalized base of support $285 \quad\left(\mathrm{~F}_{1,68}=19.76, p<0.001 ; \eta p^{2}=0.23\right)$ and percentage of double limb support $\left(\mathrm{F}_{1,68}=6.20 ; p=0.015\right.$; 
$\left.286 \eta_{p}^{2}=0.08\right)$. Mean speed, cadence, and normalized step length decreased whereas step duration,

287 normalized base of support and percentage of double limb support increased in the DT condition.

288 No other interactions or main effects were significant.

Cognitive performances. We observed lower cognitive performances in 7-9 and 10-12

291 years children as compared to adults. A main effect of age group was found for the percentage

292 and number of correct responses during the postural task $\left(\mathrm{F}_{2}, 72=3.45 ; p=0.037 ; \eta^{2}=0.09 ; \mathrm{F}_{2}\right.$,

$29372=87.32 ; p<0.001 ; \eta^{2}=0.7$, respectively) and for the percentage of correct responses during the

294 walking task $\left(\mathrm{F}_{1,68}=4.50 ; p=0.015 ; \eta^{2}=0.11\right)$. Post hoc tests indicated lower percentages of

295 correct responses in 7-9 years and 10-12 years children than in adults in the postural $(p=0.048$

296 and $p=0.025$, respectively) and the walking tasks ( $p=0.013$ and $p=0.011$, respectively). The

297 number of correct responses was lower in 7-9 years than in 10-12 years children and adults

$298(p<0.001)$ and was lower in 10-12 years children than in adults $(p<0.001)$.

Dual task cost. Our protocol also enabled the comparison between standing and walking

301 with the same cognitive task and showed that the overall DTC was higher during the walking

302 than the postural task in all groups. Statistical analysis indicated a main effect of task with higher

303 negative values during walking $\left(\mathrm{F}_{1,68}=10.40 ; p=0.002 ; \eta_{p}{ }^{2}=0.13 ;-10.72 \%\right.$ vs. $-16.5 \%$ at $7-9$

304 years; $-2.45 \%$ vs. $-10.97 \%$ at $10-12$ years; $18.71 \%$ vs. $-6.28 \%$ in adults; see Table 2 for more

305 details on all postural and walking parameters $)$. We found also a main effect of age $\left(\mathrm{F}_{2,68}=6.02\right.$;

$\left.306 p=0.004 ; \eta_{p}^{2}=0.15\right)$ with higher DTC in $7-9$ years and $10-12$ years children than in adults

$307 \quad(p=0.004$ for both comparisons).

308

309

Study 2- Dual-task effects in children with cerebral palsy 
311 degradation of standing during the DT condition whatever the age. A two-way interaction of

312 population $\mathrm{x}$ attentional condition was found for the ML mean amplitude $\left(\mathrm{F}_{1,36}=5.83 ; p=0.021\right.$;

$\left.313 \eta_{p}{ }^{2}=0.14\right)$. Post hoc analyses revealed an increase of ML mean amplitude in the CP group during

314 the DT condition $(p=0.002)$ and a greater ML amplitude in the CP than healthy children in the

315 DT $(p=0.002)$ and ST $(p=0.042)$ conditions.

316 A main effect of population was observed for the AP and ML mean amplitude $\left(\mathrm{F}_{1}\right.$,

$31736=5.69 ; \mathrm{p}=0.022 ; \eta^{2}=0.14$ and $\mathrm{F}_{1,36}=10.24 ; \mathrm{p}=0.003 ; \eta_{p}{ }^{2}=0.22$, respectively), AP and ML mean

318 speed $\left(\mathrm{F}_{1,36}=10.40 ; \mathrm{p}=0.003 ; \eta_{p}^{2}=0.22\right.$ and $\mathrm{F}_{1,36}=26.12 ; \mathrm{p}<0.001 ; \eta_{p}^{2}=0.42$, respectively $)$ and

319 sample entropy $\left(\mathrm{F}_{1,36}=6.88 ; \mathrm{p}=0.013 ; \eta p^{2}=0.16\right)$. CoP displacements were larger and sample

320 entropy was lower in CP than healthy children. A main effect of attentional condition was

321 observed for the AP and ML mean amplitude $\left(\mathrm{F}_{1,36}=6.06 ; \mathrm{p}=0.019 ; \eta_{p}{ }^{2}=0.14\right.$ and $\mathrm{F}_{1,36}=5.64$,

$\left.322 \mathrm{p}=0.023 ; \eta_{p}{ }^{2}=0.14\right)$, and AP and ML mean speed $\left(\mathrm{F}_{1,36}=17.07 ; \mathrm{p}<0.001 ; \eta_{p}{ }^{2}=0.32\right.$ and

$323 \mathrm{~F}_{1,36}=14.72 ; \mathrm{p}<0.001 ; \eta_{p}^{2}=0.29$, respectively). CoP displacements were greater in the DT

324 condition. No other interactions or main effects were significant.

Walking performances. As summarized in Figures 5 and 6, a degradation of walking

327 performances was observed, which was quite similar across populations and age groups during

328 the DT condition. The two-way interaction of age group $\mathrm{x}$ attentional condition was significant

329 for normalized step length, only $\left(\mathrm{F}_{1,38}=6.16 ; p=0.018 ; \eta^{2}=0.15\right)$ : Values were lower in the DT

330 than ST condition for CP and healthy children $(p<0.001)$.

331 A main effect of attentional task was found for mean speed $\left(\mathrm{F}_{1,38}=54.10 ; p<0.001 ; \eta_{p}^{2}\right.$

$332=0.60)$, cadence $\left(\mathrm{F}_{1,38}=19.26 ; p<0.001 ; \eta p^{2}=0.35\right)$, step duration $\left(\mathrm{F}_{1,38}=25.53 ; p<0.001 ; \eta p^{2}\right.$

$333=0.41)$, normalized step length $\left(\mathrm{F}_{1,38}=84.97 ; p<0.001 ; \eta^{2}=0.70\right)$, normalized base of support $\left(\mathrm{F}_{1}\right.$, 
$\left.33438=5.15 ; p=0.029 ; \eta p^{2}=0.13\right)$ and percentage of double limb support $\left(\mathrm{F}_{1,38}=10.37 ; p=0.003 ; \eta p^{2}\right.$

$335=0.22$ ). Mean speed, cadence, and normalized step length decreased whereas step duration,

336 normalized base of support and percentage of double limb support increased in the DT condition

337 for both populations and both age groups. No other interactions or main effects were significant. 338

Cognitive performances. We observed lower cognitive performances in 7-9 than in 10-12

340 years children and in $\mathrm{CP}$ children during the postural task, only. Main effects of age group and

341 population were found for the number of correct responses $\left(\mathrm{F}_{1,36}=13.029 ; p<0.001 ; \eta^{2}=0.27 ; \mathrm{F}_{1}\right.$,

$\left.34236=13.879 ; p<0.001 ; \eta^{2}=0.28\right)$. The number of correct responses was lower in the younger

343 children and in $\mathrm{CP}$ children. No other interactions or main effects were significant.

Dual task cost. The main effect of task was almost significant ( $p=0.059)$. The overall DTC

346 was higher during the walking than the standing task in all groups except in the 7-9 years healthy

347 children, with higher negative values during walking (-20.49\% vs. $-27.89 \%$ in $7-9$ years $\mathrm{CP}$

348 children; $-25.93 \%$ vs. $-17.46 \%$ in $7-9$ years healthy children; $-6.60 \%$ vs. $-11.94 \%$ in $10-12$ years

349 CP children; $-1.14 \%$ vs. $-19.44 \%$ in $10-12$ years healthy children; see Table 3 for more details on 350 all postural and walking parameters).

Effect of hemiplegia or diplegia. Statistical analysis revealed a two-way interaction of

353 deficit $\mathrm{x}$ attentional condition for ML mean amplitude $\left(\mathrm{F}_{1,18}=12.649 ; p=0.002 ; \eta p^{2}=0.41\right)$ and

354 sample entropy $\left(\mathrm{F}_{1,18}=10.349 ; p=0.005 ; \eta p^{2}=0.37\right)$. The interaction was almost significant for

355 AP amplitude ( $p=0.089)$. Post hoc analysis indicated that ML amplitude was significantly higher

356 in DT than in ST condition for the children with diplegia, only $(p<0.001)$. Sample entropy was

357 higher in children with hemiplegia in DT than ST condition $(p=0.005)$ and higher in children with 
358 hemiplegia than children with diplegia in the DT condition $(p=0.04)$. For the walking task, a main

359 effect of attentional condition was reported for mean speed $\left(\mathrm{F}_{1,18}=26.219 ; p<0.001 ; \eta p^{2}=0.59\right)$,

360 cadence $\left(\mathrm{F}_{1,18}=11.61 ; p=0.003 ; \eta p^{2}=0.39\right)$, step duration $\left(\mathrm{F}_{1,18}=9.59 ; p=0.006 ; \eta p^{2}=0.35\right)$,

361 percentage of double support $\left(\mathrm{F}_{1,18}=6.254 ; \mathrm{p}=0.022 ; \eta \mathrm{p}^{2}=0.26\right)$ and step length $\left(\mathrm{F}_{1,18}=35.66\right.$;

$\left.362 \mathrm{p}<0.001 ; \eta \mathrm{p}^{2}=0.66\right)$, with lower values in DT than ST for mean speed, cadence and step length.

363 The step duration and the percentage of double limb support were higher in DT. However, we

364 found no main effect of the deficit for all gait and cognitive parameters. The cognitive

365 performances were similar in children with diplegia and hemiplegia. There was only a main

366 effect of age for the number of correct responses, with lower values in younger children $\left(\mathrm{F}_{1}\right.$,

$\left.36717=5.379 ; p=0.033 ; \eta p^{2}=0.24\right)$. There was also no difference for the DTC in the postural and

368 locomotor tasks between children with hemiplegia and diplegia.

\section{Discussion}

In these two studies, we compared the standing and walking performances of healthy and

372 CP children, and adults in dual-task conditions. DTC decreased with advancing age in healthy

373 children during balance and was higher during walking in all populations. We found that adults

374 could benefit from dual-tasking for standing. Unexpectedly, the main findings indicated that CP

375 and healthy children were similarly affected by dual-task constraints during standing and

376 walking. However, children with diplegia were more affected by the DT during the postural task

377 than children with hemiplegia.

\section{Dual-task effects in healthy children and adults}

Our results confirmed that postural control becomes more efficient throughout early 
382 a decrease of postural performances in younger children (i.e., higher CoP displacements) and an

383 improvement in adults (i.e., smaller CoP displacements and higher sample entropy) under dual-

384 task constraints. CoP displacements and sample entropy were similar under single- and dual-tasks

385 in older children. There were cognitive benefits for adults but cognitive costs for younger

386 children. This suggested that standing in children aged 7-12 years is still maturating and not as

387 automated as in adults. An overall degradation of gait performances appeared in all age groups.

388 Previous studies reported a similar decrease in velocity that was associated with a decrease in

389 cadence and an increase in percentage of double limb support duration during dual-task

390 conditions for adults and children ${ }^{3}$. The fact that the DT interfered with gait control in children

391 and adults is also in accordance with the literature ${ }^{18,3}$ and confirmed that walking automaticity is

392 never complete, also in adulthood.

393 The model best explaining these different results is probably the "integrated model of task

394 prioritization" 22 . According to this model, young adults have generally intact postural reserves

395 and high hazard estimation that enable to focus on the cognitive task. The Stroop-animal task,

396 which was primarily selected for children, can be considered as a low demanding task for adults

397 in the standing condition because we observed a facilitation of the automatic control processes.

398 This easy cognitive task led to an external focus of attention and therefore to an improvement of

399 motor performances in adults, only. Children have less postural reserve than adults and the

400 cognitive task might have been too demanding especially for younger children and for the

401 walking task in all groups. Lajoie et al. ${ }^{1}$ showed that walking needed more resources than

402 standing, probably because gait requires a continuous regulation and integration of sensory

403 inputs. Our protocol confirmed that the overall DTC was higher during the walking than the

404 postural task in all groups (see Table 2). 


\section{Dual task effects in children with cerebral palsy}

Balance performances were generally weaker in $\mathrm{CP}$ than healthy children under singleand dual-tasks as illustrated by higher CoP displacements and a more regular sway. However cognitive cost of standing was quiet similar between the two populations. CP children responded similarly to healthy age-matched children to the dual-task constraints despite their pathological state. Moreover, children with diplegia showed lower postural performances in DT condition than children with hemiplegia. These latter had a higher sample entropy, reflecting an overall better postural control. These results confirmed those of Donker et al. ${ }^{45}$ and Schmit ${ }^{44}$ and were in line with the "pathological regularity versus healthy complexity" idea of Goldberger et al. ${ }^{48}$. It postulates that less "complex" or more "regular" physiological time series reflect less effective physiological control. This increased $\mathrm{CoP}$ regularity observed in $\mathrm{CP}$ children could be interpreted as the dynamical signature of an altered postural control ${ }^{45}$ and is more prominent in children with diplegia: the DTC was higher in this population and these children exhibited a more regular sway.

Interestingly and unlike previous findings, we found that $\mathrm{CP}$ children exhibited the same walking performances than healthy children whatever the single- and dual-task conditions ${ }^{49-51}$. There was no effect of the $\mathrm{CP}$ deficit on gait parameters. CP children generally exhibit reduced gait velocity, step length and increased step time and variability. A simple explanation might be that the present $\mathrm{CP}$ children aged 7-12 years with GMFCS 1 experienced standing and walking for years as healthy children: The attention needed for standing and walking was relatively the same than for healthy children and the degree of posture/gait automaticity was quite similar. Contrary to the study of Katz-Leurer et al. ${ }^{51}$, our CP participants were under Botulinum Toxin treatment since 3 to 6 months and were therefore in rather good shape to perform the various tasks. Recent findings showed that an external attentional focus promoted better balance 
430 performance for healthy and CP participants and further attenuated differences between these

431 children ${ }^{43}$. However, we did not confirm these results with the Stroop-animal task. Both single-

432 and dual-tasking showed postural deficiencies in CP children and highlighted aspects of motor

433 control that remain intact in these children. The overall DTC was higher during the walking than

434 the standing task in all groups except in the 7-9 years healthy children (see Table 3). No DTC

435 difference was observed between children with hemiplegia and diplegia, indicating that the cost

436 of postural and locomotor control was quiet similar for them.

Specific effects of the Stroop-animal task

The decline of standing and walking performances observed in all groups (except in adults

440 during standing) indicates that motor and cognitive tasks interfere with each other. Automaticity

441 depends heavily on motor task difficulty ${ }^{29}$. Our findings suggested that the development of

442 executive processes involved in the Stroop interference may proceed during healthy and

443 pathological childhood ${ }^{52}$ and/ or that healthy and CP children may require some practice of dual-

444 tasking to develop an efficient division of attentional resources and thus postural/walking

445 improvement.

446 Previous findings showed that visual tasks require the greatest attentional demand in

447 children and young adults ${ }^{3,4}$ and that there is an overlap of neural networks for balance control

448 and visual-spatial tasks ${ }^{53}$. These neural structures are still in development in children. The

449 Stroop-animal task is a discrimination and decision-making task ${ }^{4}$ requiring considerable visual

450 processing, especially in younger children, and might therefore explain the decline of their

451 postural and locomotor performances. Although Ruffieux et al. ${ }^{28}$ reported inconsistent results

452 about the effects of age on dual-tasking ability, our results showed an age-related difference

453 between healthy children and adults and a similar behavior between CP and healthy children. 
454 However, it is hard to clearly determine at which age children's performance reaches the level of

455 young adults, probably after 12 years of age. Palluel et al. ${ }^{54}$ suggested that the information

456 processing capacity is still limited in adolescents aged 14-15 years. In addition, it is reasonable to

457 assume that the age for reaching an adults' level of performance is also dependent on 1.) the type

458 and difficulty of the task and 2.) individuals' level of ability. Ruffieux et al. ${ }^{28}$ suggested that

459 tasks should be adjusted to each participant in order to obtain a comparable cognitive load in all

460 of them.

461

The integrated model of task prioritization

The simultaneous performance of two attention-demanding tasks not only causes a

464 competition for attention resources, but it also challenges the brain to decide how to prioritize the

465 two tasks. In general, prioritization may be determined by the motivation to minimize danger and

466 maximize pleasure ${ }^{22}$. It is also important to differentiate between focus of attention and

467 performance. Some subjects might change the focus of attention without performance decreases

468 until the focus has considerably shifted. Although young CP and healthy participants have lower

469 postural reserve than adults, they might have exhibited an unconscious strategy to prioritize the

470 cognitive task altering the overall motor performance because their posture and gait were not

471 challenged enough. This “posture/gait-second” strategy has already been observed during low

472 demanding standing or walking conditions ${ }^{22}$. Inhibiting a preferred response based on

473 identification of the animal's head during the Stroop-animal task may have been perceived more

474 challenging than maintaining small CoP displacements or preferred gait parameters. The hazard

475 estimation process takes into account personal limitations and may impact the prioritization to a

476 greater extent in the school-aged children ${ }^{55}$. Our results might confirm that healthy and CP

477 children are able to exhibit healthy risk judgments ${ }^{29}$. In adults, the improvement of postural 
478 control could be attributed to a stiffening strategy ${ }^{56}$ but we found less regular CoP fluctuations

479 during dual-tasking, suggesting higher degrees of freedom, a reduced attentional involvement in

480 the postural regulation and thus greater automaticity while standing ${ }^{57,58}$. The stiffening strategy

481 has already been described in children by Blanchard et al. ${ }^{59}$ but was not observed in the current

482 results. However, these authors only used conventional sway parameters for their interpretation

483 of the results (i.e., length of center of pressure path, sway range, and variability of sway).

485 Conclusion

To summarize, the main issue addressed in this experiment was to further identify

487 the attentional demand of standing and walking during typical and atypical ontogenesis. In dual-

488 task conditions, DTC decreased with age and was higher during walking in healthy children and

489 adults. CP and healthy children were similarly affected by dual-task constraints during standing

490 and walking. However, children with diplegia were more affected by the DT during the postural

491 task than children with hemiplegia. Adults could benefit from the DT during balance, only. The

492 integrated model of task prioritization might explain our results regarding postural reserve of

493 each population.

494 As already mentioned by Schmit et al. ${ }^{44}$, our study suggested that the single performance

495 of quiet stance or gait may not accurately reveal functional characteristics of standing and

496 walking in children with CP. The apparent deficits of these children might reflect different

497 strategies or adaptations because simultaneous execution of motor and cognitive tasks is

498 embedded in the daily life of every child. Examining postural control and gait in the context of

499 dual-tasking exposes aspects of motor control that may remain intact in CP children (e.g., the

500 ability to adapt standing/gait to perform a visual task) and other aspects that may be disrupted

501 (e.g., the ability to allocate attention in such a way as to enhance performance). It is also worth to 
502 mention that children within the same mobility classification level show an evident and consistent

503 pattern of differences. For example, children with hemiplegia clearly show better gait and lower

504 extremity mobility scores when compared to those with diplegia ${ }^{60}$. Considering standing and gait

505 within a framework of functional behavior seems to be crucial in this population. 


\section{References}

1 Lajoie Y, Teasdale N, Bard C, Fleury M. Attention demands for static and dynamic equilibriuum. Experimental Brain Research 1993; 97: 139-44.

2 Pellecchia GL. Postural sway increases with attentional demands of concurrent cognitive task. Gait and Posture 2003; 18: 29-34.

3 Chauvel G, Palluel E, Brandao A, Barbieri G, Nougier V, Olivier I. Attentional load of walking in children aged 7-12 and in adults. Gait Posture 2017; 56: 95-9.

4 Al-Yahya E, Dawes H, Smith L, Dennis A, Howells K, Cockburn J. Cognitive motor interference while walking: a systematic review and meta-analysis. Neurosci Biobehav Rev 2011; 35: 715-28.

5 Woollacott M, Shumway-Cook A. Attention and the control of posture and gait: a review of an emerging area of research. Gait Posture 2002; 16: 1-14.

6 Huxhold O, Li SC, Schmiedek F, Lindenberger U. Dual-tasking postural control: aging and the effects of cognitive demand in conjunction with focus of attention. Brain Res Bull 2006; 69: 294-305.

7 Polskaia N, Lajoie Y. Reducing postural sway by concurrently performing challenging cognitive tasks. Hum Mov Sci 2016; 46: 177-83.

8 Olivier I, Palluel E, Nougier V. Effects of attentional focus on postural sway in children and adults. Exp Brain Res 2008; 185: 341-5.

9 Richer N, Saunders D, Polskaia N, Lajoie Y. The effects of attentional focus and cognitive tasks on postural sway may be the result of automaticity. Gait Posture 2017; 54: 45-9.

10 Wulf G, McNevin NH, Shea $\mathrm{CH}$. The automaticity of complex motor skill learning as a function of attentional focus. The Quarterly Journal of Experimental Psychology. A: Human Experimental Psychology 2001; 54: 1143-54.

11 Verrel J, Lovden M, Schellenbach M, Schaefer S, Lindenberger U. Interacting effects of cognitive load and adult age on the regularity of whole-body motion during treadmill walking. Psychology and aging 2009; 24: 75-81.

12 Kerr B, Condon S, McDonald L. Cognitive spatial processing and the regulation of posture. Journal of Experimental Psychology. Human Perception and Performance 1985; 11: 617-22.

13 Remaud A, Boyas S, Caron GA, Bilodeau M. Attentional demands associated with postural control depend on task difficulty and visual condition. J Mot Behav 2012; 44: 329-40.

14 Dault MC, Geurts AC, Mulder TW, Duysens J. Postural control and cognitive task performance in healthy participants while balancing on different support-surface configurations. Gait Posture 2001; 14: 248-55.

15 Woollacott M, Vander Velde T. Non-visual spatial tasks reveal increased interactions with stance postural control. Brain Res 2008; 1208: 95-102.

16 Lin MI, Lin KH. Walking while Performing Working Memory Tasks Changes the Prefrontal Cortex Hemodynamic Activations and Gait Kinematics. Frontiers in behavioral neuroscience 2016; 10: 92.

17 Lacour M, Bernard-Demanze L, Dumitrescu M. Posture control, aging, and attention resources: models and posture-analysis methods. Neurophysiol Clin 2008; 38: 411-21.

18 Patel P, Lamar M, Bhatt T. Effect of type of cognitive task and walking speed on cognitivemotor interference during dual-task walking. Neuroscience 2014; 260: 140-8.

19 Luder B, Kiss R, Granacher U. Single- and Dual-Task Balance Training Are Equally Effective in Youth. Frontiers in psychology 2018; 9: 912. 
551

552

553

554

555

556

557

558

559

560

561

562

563

564

565

566

567

568

569

570

571

572

573

574

575

576

577

578

579

580

581

582

583

584

585

586

587

588

589

590

591

592

593

594

595

596

597

20 Shumway-Cook A, Woollacott M, Kerns KA, Baldwin M. The effects of two types of cognitive tasks on postural stability in older adults with and without a history of falls. $J$ Gerontol A Biol Sci Med Sci 1997; 52: M232-40.

21 Andersson G, Hagman J, Talianzadeh R, Svedberg A, Larsen HC. Dual-task study of cognitive and postural interference in patients with vestibular disorders. Otol Neurotol 2003; 24: 289-93.

22 Yogev-Seligmann G, Hausdorff JM, Giladi N. Do we always prioritize balance when walking? Towards an integrated model of task prioritization. Movement disorders : official journal of the Movement Disorder Society 2012; 27: 765-70.

23 Olivier I, Cuisinier R, Vaugoyeau M, Nougier V, Assaiante C. Dual-task study of cognitive and postural interference in 7-year-olds and adults. Neuroreport 2007; 18: 817-21.

24 Rival C, Ceyte H, Olivier I. Developmental changes of static standing balance in children. Neuroscience Letters 2005; 376: 133-6.

25 Sutherland DH, Olshen R, Cooper L, Woo SL. The development of mature gait. $J$ Bone Joint Surg Am 1980; 62: 336-53.

26 Kraan CM, Tan AHJ, Cornish KM. The developmental dynamics of gait maturation with a focus on spatiotemporal measures. Gait Posture 2017; 51: 208-17.

27 Hausdorff JM, Zemany L, Peng C, Goldberger AL. Maturation of gait dynamics: stride-tostride variability and its temporal organization in children. $J$ Appl Physiol (1985) 1999; 86: 10407.

28 Ruffieux J, Keller M, Lauber B, Taube W. Changes in Standing and Walking Performance Under Dual-Task Conditions Across the Lifespan. Sports Med 2015; 45: 1739-58.

29 Schott N, Klotzbier TJ. Profiles of Cognitive-Motor Interference During Walking in Children: Does the Motor or the Cognitive Task Matter? Frontiers in psychology 2018; 9: 947.

30 Saxena S, Cinar E, Majnemer A, Gagnon I. Does dual tasking ability change with age across childhood and adolescence? A systematic scoping review. International journal of developmental neuroscience : the official journal of the International Society for Developmental Neuroscience 2017; 58: 35-49.

31 Schaefer S, Jagenow D, Verrel J, Lindenberger U. The influence of cognitive load and walking speed on gait regularity in children and young adults. Gait Posture 2015; 41: 258-62.

32 Hagmann-von Arx P, Manicolo O, Lemola S, Grob A. Walking in School-Aged Children in a Dual-Task Paradigm Is Related to Age But Not to Cognition, Motor Behavior, Injuries, or Psychosocial Functioning. Frontiers in psychology 2016; 7: 352.

33 Boonyong S, Siu KC, van Donkelaar P, Chou LS, Woollacott MH. Development of postural control during gait in typically developing children: the effects of dual-task conditions. Gait Posture 2012; 35: 428-34.

34 Woollacott M, Shumway-Cook A. Postural dysfunction during standing and walking in children with cerebral palsy: what are the underlying problems and what new therapies might improve balance? Neural plasticity 2005; 12: 211-9; discussion 63-72.

35 Ferrari A, Bergamini L, Guerzoni G, Calderara S, Bicocchi N, Vitetta G, Borghi C, Neviani R. Gait-Based Diplegia Classification Using LSMT Networks. Journal of healthcare engineering 2019; 2019: 3796898 .

36 Wren TA, Rethlefsen S, Kay RM. Prevalence of specific gait abnormalities in children with cerebral palsy: influence of cerebral palsy subtype, age, and previous surgery. $J$ Pediatr Orthop 2005; 25: 79-83.

37 Bell KJ, Ounpuu S, DeLuca PA, Romness MJ. Natural progression of gait in children with cerebral palsy. J Pediatr Orthop 2002; 22: 677-82. 
598

599

600

601

602

603

604

605

606

607

608

609

610

611

612

613

614

615

616

617

618

619

620

621

622

623

624

625

626

627

628

629

630

631

632

633

634

635

636

637

638

639

640

641

642

643

644

38 Johnson DC, Damiano DL, Abel MF. The evolution of gait in childhood and adolescent cerebral palsy. J Pediatr Orthop 1997; 17: 392-6.

39 Galli M, Cimolin V, Rigoldi C, Tenore N, Albertini G. Gait patterns in hemiplegic children with Cerebral Palsy: comparison of right and left hemiplegia. Research in developmental disabilities 2010; 31: 1340-5.

40 Ickx G, Hatem SM, Riquelme I, Friel KM, Henne C, Araneda R, Gordon AM, Bleyenheuft Y. Impairments of Visuospatial Attention in Children with Unilateral Spastic Cerebral Palsy. Neural plasticity 2018; 2018: 1435808.

41 Reilly DS, Woollacott MH, van Donkelaar P, Saavedra S. The interaction between executive attention and postural control in dual-task conditions: children with cerebral palsy. Arch Phys Med Rehabil 2008; 89: 834-42.

42 Hung YC, Meredith GS. Influence of dual task constraints on gait performance and bimanual coordination during walking in children with unilateral cerebral palsy. Research in developmental disabilities 2014; 35: 755-60.

43 Schmit J, Riley M, Cummins-Sebree S, Schmitt L, Shockley K. Functional Task Constraints Foster Enhanced Postural Control in Children With Cerebral Palsy. Physical therapy 2016; 96: 348-54.

44 Schmit J, Riley M, Cummins-Sebree S, Schmitt L, Shockley K. Children with cerebral palsy effectively modulate postural control to perform a supra-postural task. Gait Posture 2015; 42: 4953.

45 Donker SF, Ledebt A, Roerdink M, Savelsbergh GJ, Beek PJ. Children with cerebral palsy exhibit greater and more regular postural sway than typically developing children. Exp Brain Res 2008; 184: 363-70.

46 Richman JS, Moorman JR. Physiological time-series analysis using approximate entropy and sample entropy. American journal of physiology. Heart and circulatory physiology 2000; 278: H2039-49.

47 Wright I, Waterman M, Prescott H, Murdoch-Eaton D. A new Stroop-like measure of inhibitory function development: typical developmental trends. Journal of child psychology and psychiatry, and allied disciplines 2003; 44: 561-75.

48 Goldberger AL, Amaral LA, Hausdorff JM, Ivanov P, Peng CK, Stanley HE. Fractal dynamics in physiology: alterations with disease and aging. Proc Natl Acad Sci U S A 2002; 99 Suppl 1: 2466-72.

49 Hsue BJ, Miller F, Su FC. The dynamic balance of the children with cerebral palsy and typical developing during gait. Part I: Spatial relationship between COM and COP trajectories. Gait Posture 2009; 29: 465-70.

50 Hsue BJ, Miller F, Su FC. The dynamic balance of the children with cerebral palsy and typical developing during gait Part II: Instantaneous velocity and acceleration of COM and COP and their relationship. Gait Posture 2009; 29: 471-6.

51 Katz-Leurer M, Rotem H, Meyer S. Effect of concurrent cognitive tasks on temporo-spatial parameters of gait among children with cerebral palsy and typically developed controls. Developmental neurorehabilitation 2014; 17: 363-7.

52 Adleman NE, Menon V, Blasey CM, White CD, Warsofsky IS, Glover GH, Reiss AL. A developmental fMRI study of the Stroop color-word task. NeuroImage 2002; 16: 61-75.

53 Barra J, Bray A, Sahni V, Golding JF, Gresty MA. Increasing cognitive load with increasing balance challenge: recipe for catastrophe. Exp Brain Res 2006; 174: 734-45.

54 Palluel E, Nougier V, Olivier I. Postural control and attentional demand during adolescence. Brain Res 2010; 1358: 151-9. 
64555 Abbruzzese LD, Rao AK, Bellows R, Figueroa K, Levy J, Lim E, Puccio L. Effects of 646 manual task complexity on gait parameters in school-aged children and adults. Gait Posture 2014; 647 40: 658-63.

$64856 \quad$ McNevin NH, Wulf G. Attentional focus on supra-postural tasks affects postural control. 649 Hum Mov Sci 2002; 21: 187-202.

65057 Stins JF, Roerdink M, Beek PJ. To freeze or not to freeze? Affective and cognitive 651 perturbations have markedly different effects on postural control. Human Movement Science 2011; 652 30: 190-202.

65358 Donker SF, Roerdink M, Greven AJ, Beek PJ. Regularity of center-of-pressure trajectories 654 depends on the amount of attention invested in postural control. Exp Brain Res 2007; 181: 1-11.

65559 Blanchard Y, Carey S, Coffey J, Cohen A, Harris T, Michlik S, Pellecchia GL. The 656 influence of concurrent cognitive tasks on postural sway in children. Pediatr Phys Ther 2005; 17: $657 \quad 189-93$.

65860 Damiano D, Abel M, Romness M, Oeffinger D, Tylkowski C, Gorton G, Bagley A, 659 Nicholson D, Barnes D, Calmes J, Kryscio R, Rogers S. Comparing functional profiles of children 660 with hemiplegic and diplegic cerebral palsy in GMFCS Levels I and II: Are separate classifications 661 needed? Dev Med Child Neurol 2006; 48: 797-803.

662

663 


\section{FIGURE CAPTION}

665

666 Figure 1: Mean performance and standard deviation for the five postural parameters (AP/ML mean amplitude and speed, sample entropy (SampEn)) and for the two conditions (ST, DT) as a function of age group (7-9 years, 10-12 years, and adults). SampEn combines AP and ML axes. For readability purposes, significant differences between age groups were not drawn on the figure (please refer to text for details). ${ }^{*} \mathrm{p}<0.05$ and ${ }^{*} \mathrm{p}<0.01$

Figure 2: Mean performance and standard deviation for the six gait parameters (mean speed, cadence, step duration, normalized step length, normalized base of support and percentage of double limb support) and for the two conditions (ST, DT) as a function of age group (7-9 years, 10-12 years, and adults). $* \mathrm{p}<0.05$ and $* * \mathrm{p}<0.01$

Figures 3 and 4: Mean performance and standard deviation for the five postural parameters (AP/ML mean amplitude and speed, sample entropy (SampEn)) and for the two conditions (ST, DT) as a function of population (CP, healthy children) and age group (7-9 years, 10-12 years). SampEn combines AP and ML axes. $* \mathrm{p}<0.05$ and $* * p<0.01$

Figures 5 and 6: Mean performance and standard deviation for the six gait parameters (mean speed, cadence, step duration, normalized step length, normalized base of support and percentage of double limb support) and for the two conditions (ST, DT) as a function of population (CP, healthy children) and age group (7-9 years, $10-12$ years). ${ }^{*} \mathrm{p}<0.05$ and $* * \mathrm{p}<0.01$ 


\section{TABLES}

689 Table 1. Characteristics of the sample for study 2

\begin{tabular}{lrr} 
& CP $(\mathrm{n})$ & TD $(\mathrm{n})$ \\
\hline Total & 20 & 20 \\
& & \\
Sex & & \\
$\quad$ Female & 7 & 7 \\
$\quad$ Male & 13 & 13
\end{tabular}

Distribution of cerebral palsy

$\begin{array}{lrr}\text { Diplegia } & 11 & \text { N/A } \\ \text { Hemiplegia } & 9 & \end{array}$

Gross Motor Functional

Classification

I

$20 \quad$ N/A

Orthotic use

Yes 0 N/A

No 20

690

691

692 CP, cerebral palsy; TD, typically developing. 
693 Table 2. DTC for standing and walking tasks in healthy children and adults (study 1). Values

694 represent means and standard deviation (SD).

695

\begin{tabular}{|c|c|c|c|c|c|c|}
\hline & \multicolumn{2}{|c|}{$7-9$ years } & \multicolumn{2}{|c|}{$10-12$ years } & \multicolumn{2}{|c|}{ Adults } \\
\hline & Mean & SD & Mean & SD & Mean & SD \\
\hline \multicolumn{7}{|c|}{$\begin{array}{l}\text { Standing performances (DTC in } \\
\%)\end{array}$} \\
\hline AP mean amplitude & $-18,41$ & 59,02 & $-3,39$ & 32,80 & 13,08 & 29,88 \\
\hline ML mean amplitude & $-7,81$ & 41,06 & $-9,61$ & 45,63 & 20,49 & 33,42 \\
\hline AP mean speed & $-28,54$ & 54,39 & $-16,39$ & 13,58 & $-8,76$ & 18,91 \\
\hline ML mean speed & $-20,44$ & 62,46 & $-6,81$ & 18,78 & 1,79 & 21,23 \\
\hline Sample Entropy & 21,60 & 67,33 & 23,93 & 49,90 & 66,97 & 76,12 \\
\hline Mean DTC & $-10,72$ & 56,85 & $-2,45$ & 32,14 & 18,71 & 35,91 \\
\hline \multicolumn{7}{|c|}{ Walking performances (DTC in \%) } \\
\hline Mean speed & $-19,01$ & 15,04 & $-15,44$ & 9,51 & $-10,43$ & 8,10 \\
\hline Cadence & $-7,06$ & 12,23 & $-4,71$ & 6,49 & $-3,53$ & 5,90 \\
\hline Step duration & $-15,36$ & 30,01 & $-5,24$ & 7,33 & $-4,14$ & 6,83 \\
\hline Normalized step length & $-13,35$ & 7,13 & $-11,61$ & 7,91 & $-7,33$ & 4,17 \\
\hline Normalized base of support & $-34,11$ & 48,84 & $-19,10$ & 41,91 & $-5,42$ & 16,89 \\
\hline Double limb support & $-10,11$ & 19,42 & $-9,71$ & 10,47 & $-6,84$ & 10,82 \\
\hline Mean DTC & $-16,50$ & 22,11 & $-10,97$ & 13,94 & $-6,28$ & 8,79 \\
\hline
\end{tabular}


Table 3. DTC for standing and walking tasks in CP and healthy children (study 2). Values represent means and standard deviation (SD).

\section{Children with CP Healthy children}

\begin{tabular}{|c|c|c|c|c|c|c|c|c|}
\hline & \multicolumn{2}{|c|}{$7-9$ years } & \multicolumn{2}{|c|}{$10-12$ years } & \multicolumn{2}{|c|}{$7-9$ years } & \multicolumn{2}{|c|}{$10-12$ years } \\
\hline & Mean & SD & Mean & SD & Mean & SD & Mean & SD \\
\hline \multicolumn{9}{|c|}{ Standing performances (DTC in \%) } \\
\hline AP mean amplitude & $-19,35$ & 28,90 & $-14,75$ & 32,83 & $-41,90$ & 87,41 & $-8,03$ & 36,97 \\
\hline ML mean amplitude & $-24,05$ & 30,18 & $-32,72$ & 49,56 & $-14,19$ & 62,37 & 7,80 & 17,11 \\
\hline AP mean speed & $-20,29$ & 14,54 & $-10,94$ & 14,90 & $-44,57$ & 83,39 & $-19,70$ & 9,76 \\
\hline ML mean speed & $-25,79$ & 16,46 & $-16,35$ & 19,59 & $-38,53$ & 97,92 & $-1,92$ & 22,33 \\
\hline Sample Entropy & $-12,96$ & 34,19 & 41,76 & 89,44 & 9,53 & 64,65 & 16,15 & 52,38 \\
\hline Mean DTC & $-20,49$ & 24,85 & $-6,60$ & 41,26 & $-25,93$ & 79,15 & $-1,14$ & 27,71 \\
\hline \multicolumn{9}{|c|}{ Walking performances (DTC in \%) } \\
\hline Mean speed & $-22,43$ & 14,56 & $-16,12$ & 18,37 & $-25,32$ & 18,67 & $-24,93$ & 16,19 \\
\hline Cadence & $-10,04$ & 11,14 & $-7,54$ & 12,54 & $-11,98$ & 14,88 & $-9,52$ & 14,30 \\
\hline Step duration & $-12,60$ & 15,67 & $-11,12$ & 17,15 & $-10,85$ & 9,19 & $-6,53$ & 4,58 \\
\hline Normalized step length & $-10,70$ & 7,19 & $-10,87$ & 8,96 & $-16,73$ & 8,61 & $-18,05$ & 10,61 \\
\hline Normalized base of support & $-89,59$ & 61,56 & $-4,50$ & 34,37 & $-29,23$ & 37,21 & $-49,11$ & 67,88 \\
\hline Double limb support & $-22,00$ & 20,57 & $-21,49$ & 57,17 & $-10,63$ & 7,85 & $-8,53$ & 14,15 \\
\hline Mean DTC & $-27,89$ & 21,78 & $-11,94$ & 24,76 & $-17,46$ & 16,07 & $-19,44$ & 21,28 \\
\hline
\end{tabular}


